\title{
A Study on Some Special Forms of Holographic Ricci Dark Energy in Fractal Universe
}

\author{
Surajit Chattopadhyay, ${ }^{1}$ Antonio Pasqua, ${ }^{2}$ and Sudipto Roy $^{3}$ \\ ${ }^{1}$ Mathematics Section, Department of Computer Application, Pailan College of Management and Technology, Sector-1 Phase I, \\ Bengal Pailan Park (off D.H. Road), Joka, Kolkata 700 104, India \\ ${ }^{2}$ Department of Physics, University of Trieste, Via Valerio 2, 34100 Trieste, Italy \\ ${ }^{3}$ Department of Physics, St. Xavier's College, 30 Park Street (30 Mother Teresa Sarani), Kolkata 700 016, India
}

Correspondence should be addressed to Surajit Chattopadhyay; surajitchatto@gmail.com

Received 26 August 2013; Accepted 15 September 2013

Academic Editors: C. Ahn and C. A. De. S. Pires

Copyright (C) 2013 Surajit Chattopadhyay et al. This is an open access article distributed under the Creative Commons Attribution License, which permits unrestricted use, distribution, and reproduction in any medium, provided the original work is properly cited.

We have considered the modified and the extended holographic Ricci dark energy models (MHRDE and EHRDE) in fractal universe. We have assumed a time-like fractal profile $v=t^{-\beta}$, where $\beta=4(1-\alpha)$. We have reconstructed the Hubble parameter $H$, the energy density, the equations of state parameter $w$, and the deceleration parameter $q$ for both MHRDE and EHRDE.

\section{Introduction}

Motivated by the virtues and problems of Horava-Lifshitz gravity, Calcagni [1] formulated an effective quantum field theory with two key features: (i) power-counting renormalizability is obtained when the fractal behaviour is realized at structural level; (ii) Lorentz invariance is maintained. Calcagni [2] proposed a field theory which lives in fractal space-time and argued this to be Lorentz invariant, powercounting renormalizable, ultraviolet finite, and causal and discussed its implications for quantum gravity, cosmology, and the cosmological constant. Lemets and Yerokhin [3] demonstrated the key features and motivations of fractal cosmology models. Sheykhi et al. [4] investigated the thermodynamical properties of the apparent horizon in a fractal universe. Karami et al. [5] investigated the holographic, the new agegraphic, and the ghost dark energy models in the framework of fractal cosmology, and they also discussed the behavior of the equations of state parameter $\omega$. In the present work, we are going to investigate the behavior of the modified holographic Ricci dark energy (MHRDE) and the extended holographic Ricci dark energy (EHRDE) models in fractal universe.
The holographic dark energy (HDE) model, proposed by Li [6], is inspired by the holographic principle [7], which states that the maximum number of degrees of freedom in a volume should be proportional to the surface area [8]. Applying the novel principle that $L^{3} \rho_{\Lambda} \leq L M_{P}^{2}$ (where the infrared (IR) cut-off is encoded by the scale $L, \rho_{\Lambda}$ corresponds to the quantum zero-point energy density, and $M_{P}^{-2}=8 \pi G$ represents the Planck mass, with $G$ being the Newton's gravitational constant) within the cosmological context implies that the dark energy density of the universe $\rho_{x}$ takes the same form as the vacuum energy; that is, $\rho_{\Lambda}=$ $\rho_{x}$ [8]. Using the largest $L$ as the one saturating the above inequality, it turns out to be HDE, whose density is given by $\rho_{\Lambda}=3 c^{2} M_{p}^{2} L^{-2}[6]$, where $c$ is a numerical factor. Many IR cut-offs have been considered in recent works, for example, the large scale of the universe, the Hubble horizon, the particle horizon, the event horizon or generalized IR cutoff, and so forth [8-12]. Studies on HDE from various points of view include [13-18]. Gao et al. [19] have proposed a HDE model, where the future event horizon is replaced by the inverse of the Ricci scalar curvature, and this model is named as "Ricci dark energy model" (RDE). This model does not only avoid the causality problem and is phenomenologically 
viable, but also solve the coincidence problem of dark energy [20]. The Ricci curvature of FRW universe is given by $R=$ $-6\left(\dot{H}+2 H^{2}+k / a^{2}\right)$. The RDE proposed in [19] has energy density which is proportional to the Ricci scalar curvature; that is, $\rho_{\Lambda} \propto R$. Some significant works on RDE are [21-31]. In the present work, we are considering two special modified forms of holographic RDE:

(i) modified holographic Ricci dark energy (MHRDE) $[8,10,32,33]$, whose energy density is given by $\rho_{\text {MHRDE }}=(2 /(\xi-\eta))\left(\dot{H}+(3 \xi / 2) H^{2}\right)$, where $\xi$ and $\eta$ are free constants;

(ii) extended holographic Ricci dark energy (EHRDE) $[10,34]$, whose density has the form $\rho_{\mathrm{EHRDE}}=$ $3 M_{p}^{2}\left(\xi H^{2}+\eta \dot{H}\right)$, where $M_{p}^{2}$ is the reduced Planck mass and $\xi$ and $\eta$ are constants to be determined. If $\xi=2 \eta$, then we get back to RDE.

In the present work, we will consider separately that the fractal universe is filled with MHRDE and EHRDE, respectively. We will reconstruct the Hubble parameter $H$ for both cases. Based on the reconstructed Hubble parameters, we will investigate the behavior of the equation of state parameter $\omega$ and the deceleration parameter $q$.

\section{The Dark Energy Models in the Fractal Universe}

The total action of Einstein gravity in a fractal space-time is given by [4]

$$
S=S_{G}+S_{m}
$$

where the gravitational part of the action is given by

$$
S_{G}=\frac{1}{16 \pi G} \int d \varrho(x) \sqrt{-g}\left(R-2 \Lambda-\omega \partial_{\mu} v \partial^{\mu} v\right),
$$

and the matter part of the action is given by

$$
S_{m}=\int d \varrho(x) \sqrt{-g} \mathscr{L}_{m} .
$$

Here, $g$ is the determinant of the dimensionless metric $g_{\mu \nu}$, $\Lambda$ and $R$ are, respectively, the cosmological constant and the Ricci scalar. Moreover, $v$ is the fractional function, and $\omega$ is the fractal parameter. The standard measure $d^{4} x$ is replaced with a Lebesgue-Stieltjes measure $d \varrho(x)$. Taking the variation of the action given in (1) with respect to the FRW metric $g_{\mu \nu}$, we obtain the Friedmann equations in a fractal universe as [4]

$$
\begin{gathered}
H^{2}+\frac{k}{a^{2}}+H \frac{\dot{v}}{v}-\frac{\omega}{6} \dot{v}^{2}=\frac{8 \pi G}{3} \rho+\frac{\Lambda}{3}, \\
\dot{H}+H^{2}-H \frac{\dot{v}}{v}+\frac{\omega}{3} \dot{v}^{2}-\frac{1}{2} \frac{\square v}{v}=-\frac{8 \pi G}{6}(\rho+3 p)+\frac{\Lambda}{3} .
\end{gathered}
$$

The curvature parameter $k=0,1,-1$ corresponds to a flat, closed, and open universe, respectively. In the present work, we will consider $k=0$ as flat universe. The continuity equation in a fractal universe takes the form

$$
\dot{\rho}+\left(3 H+\frac{\dot{v}}{v}\right)(\rho+p)=0 .
$$

It is clear that for $v=1$, the standard Friedmann equations are recovered. We further assume that only the time direction is fractal while spatial slices have usual geometry. Assuming a time-like fractal profile $v=t^{-\beta}$, where $\beta=4(1-\alpha)$ (with $(0<$ $\alpha \leq 1)$ ) is the fractal dimension. The Friedmann equations given in (4) in the absence of the cosmological constant can be written as

$$
\begin{gathered}
H^{2}+\frac{k}{a^{2}}+H \frac{\beta}{t}-\frac{\omega \beta^{2}}{6 t^{2(\beta+1)}}=\frac{8 \pi G}{3} \rho, \\
\dot{H}+H^{2}-H \frac{\beta}{2 t}+\frac{\beta(\beta+1)}{2 t^{2}}+\frac{\omega \beta^{2}}{3 t^{2(\beta+1)}}=-\frac{8 \pi G}{6}(\rho+3 p),
\end{gathered}
$$

while the continuity equation given in (5) takes the form

$$
\dot{\rho}+\left(3 H-\frac{\beta}{t}\right)(\rho+p)=0
$$

In (6), we replace $\rho$ by $\rho_{\mathrm{MHRDE}}$ and $\rho_{\mathrm{EHRDE}}$, respectively, for flat FRW universe $(k=0)$, and we get nonlinear differential equations on $H$. For MHRDE, we have

$$
\dot{\rho}_{\mathrm{MHRDE}}=\frac{2}{\xi-\eta}(3 \xi H \dot{H}+\ddot{H}) .
$$

Using (9) in continuity equation given in (8), we get the pressure $p_{\mathrm{MHRDE}}$ for the MHRDE model as follows:

$$
p_{\text {MHRDE }}=\frac{2}{\xi-\eta}\left[-\frac{3}{2} \xi H^{2}-\dot{H}+\frac{t}{\beta-3 t H}(3 \xi H \dot{H}+\ddot{H})\right] .
$$

For EHRDE we have

$$
\dot{\rho}_{\mathrm{EHRDE}}=6 \xi H \dot{H}+3 \eta \ddot{H} \text {. }
$$

Using (11) in continuity equation given in (8), we get the pressure $p_{\mathrm{EHRDE}}$ for the EHRDE model as follows:

$$
p_{\text {EHRDE }}=-3\left(\xi H^{2}+\eta \dot{H}\right)+\frac{3 t(2 \xi H \dot{H}+\eta \ddot{H})}{\beta-3 t H} .
$$

Since we cannot have analytical solution of the differential equations, we solve them numerically.

We plot the reconstructed Hubble parameter $\widetilde{H}$ in Figures 1 and 2 for MHRDE and EHRDE, respectively. In the figures for MHRDE the solid, dashed, and dot-dashed lines correspond to $(\xi=0.5, \eta=1.12),(\xi=0.5, \eta=1.14)$, and $(\xi=0.5, \eta=1.17)$, respectively. For EHRDE, the solid, dashed, and dot-dashed lines correspond to $(\xi=1, \eta=2)$, $(\xi=1.01, \eta=2)$, and $(\xi=1.05, \eta=2.01)$, respectively. For all figures, we have considered $\alpha=0.8$ and $\omega=0.02$. 


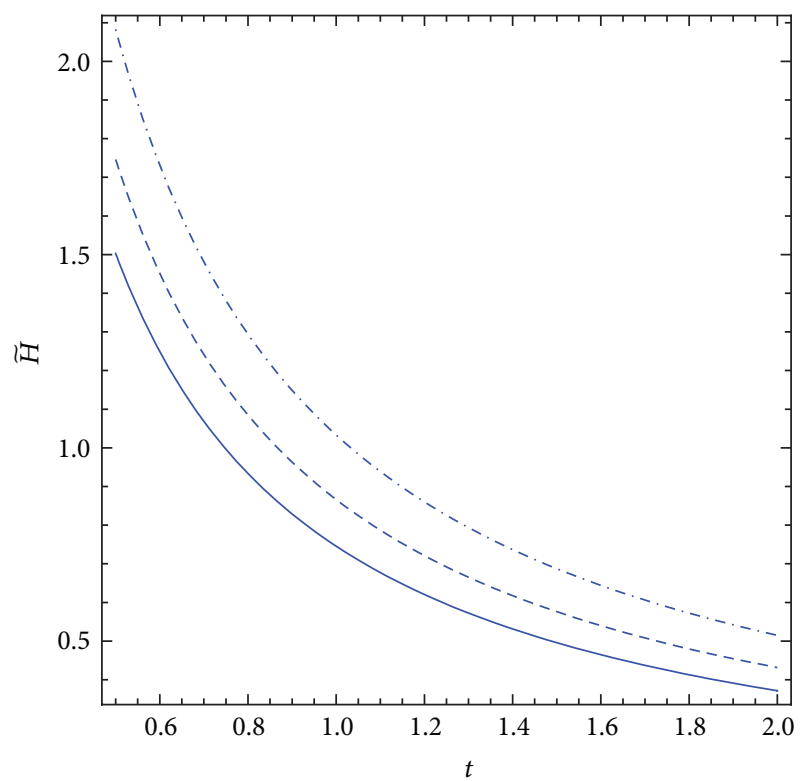

FIGURE 1: Reconstructed Hubble parameter $\widetilde{H}$ for MHRDE in fractal universe.

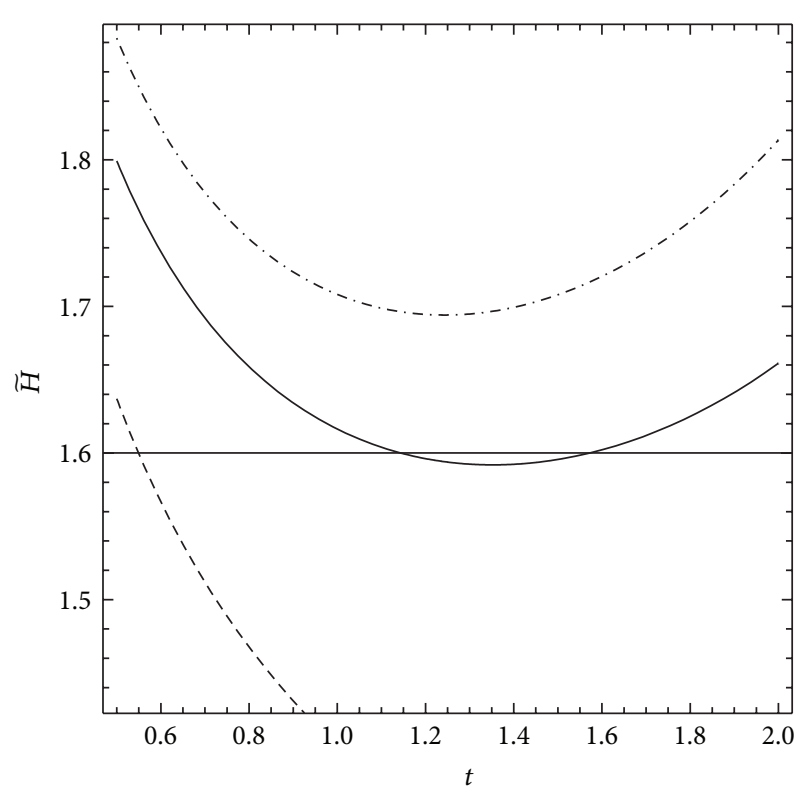

FIGURE 2: Reconstructed Hubble parameter $\widetilde{H}$ for EHRDE in fractal universe.

In Figure 1, we observe that $\widetilde{H}$ is a decreasing function of cosmic time $t$ for MHRDE. In Figure 2, we observe that for $(\xi=1.01, \eta=2)$ in EHRDE, $\widetilde{H}$ is a decreasing function of $t$. However, for the other combinations, $\widetilde{H}$ is decreasing up to $t \approx 1.2$, and then it is increasing gradually. Next we plot the time derivatives of the reconstructed Hubble parameter $\dot{\widetilde{H}}$ in Figures 3 and 4. For MHRDE (Figure 3), we find that $\dot{\widetilde{H}}$ is increasing with $t$ and staying at negative level throughout. Although for EHRDE (Figure 4) we observe that

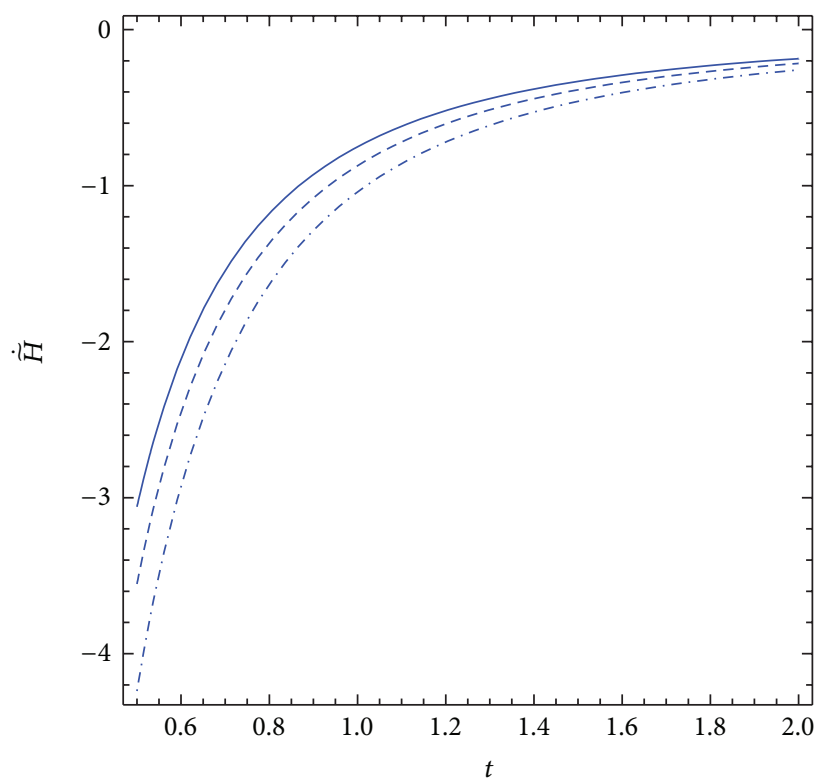

FIgURE 3: Time derivative of reconstructed Hubble parameter $\dot{\vec{H}}$ for MHRDE in fractal universe.

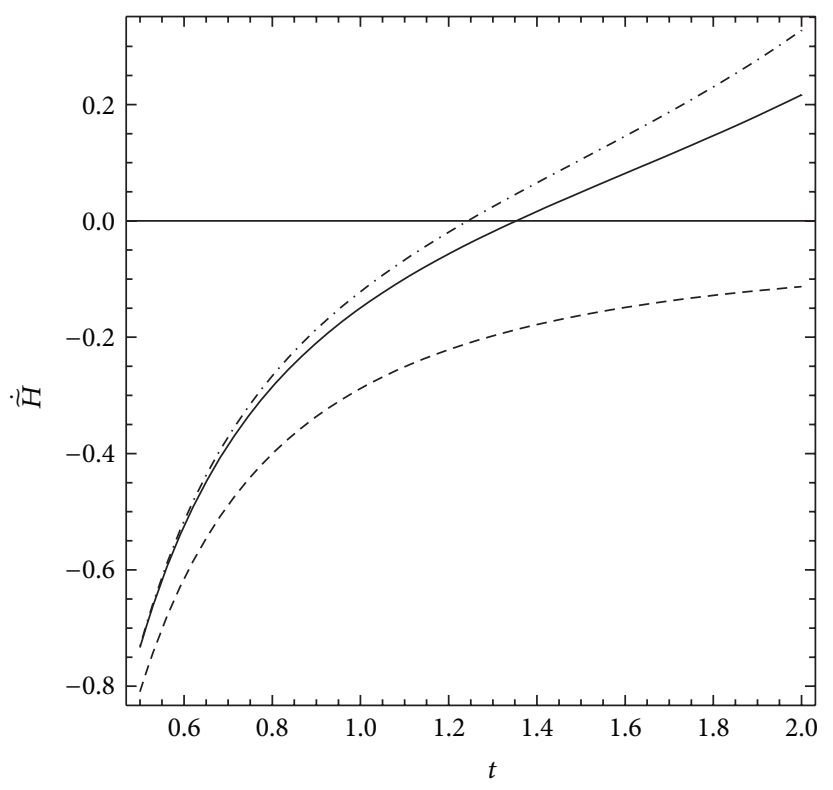

FIGURE 4: Time derivative of reconstructed Hubble parameter $\dot{\widetilde{H}}$ for EHRDE in fractal universe.

$\dot{\widetilde{H}}$ is increasing with $t$, we find that for $(\xi=1, \eta=2)$ and $(\xi=1.05, \eta=2.01)$ the $\dot{\vec{H}}$ is changing its sign at $t \approx 1.3$.

In Figure 5, we find that $\rho_{\mathrm{MHRDE}}$ is decreasing with $t$. Instead, in Figure 6 , we observe that $\rho_{\mathrm{EHRDE}}$ is increasing with $t$. The rate of increasing is less for $(\xi=1.01, \eta=2)$ than for the other combinations. The negative pressure for MHRDE is decreasing with time as shown in Figure 7. Instead, for EHRDE, the negative pressure is increasing for $(\xi=1, \eta=2)$ and $(\xi=1.05, \eta=2.01)$ (Figure 8). The EoS parameters $w_{\mathrm{MHRDE}}=p_{\mathrm{MHRDE}} / \rho_{\mathrm{MHRDE}}$ and $w_{\mathrm{EHRDE}}=p_{\mathrm{EHRDE}} / \rho_{\mathrm{EHRDE}}$ 


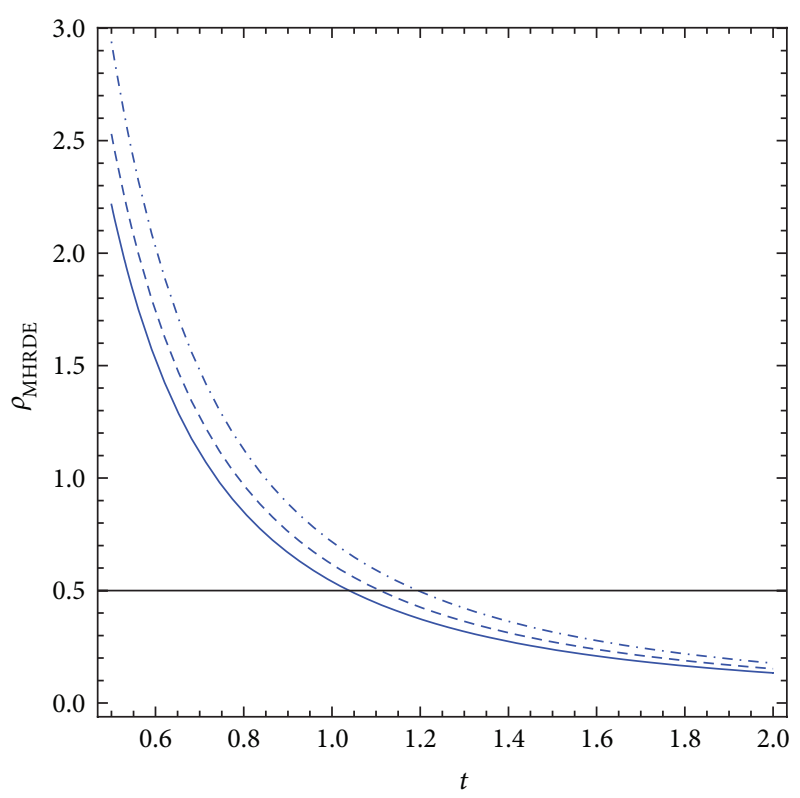

FIGURE 5: Density of MHRDE in fractal universe.

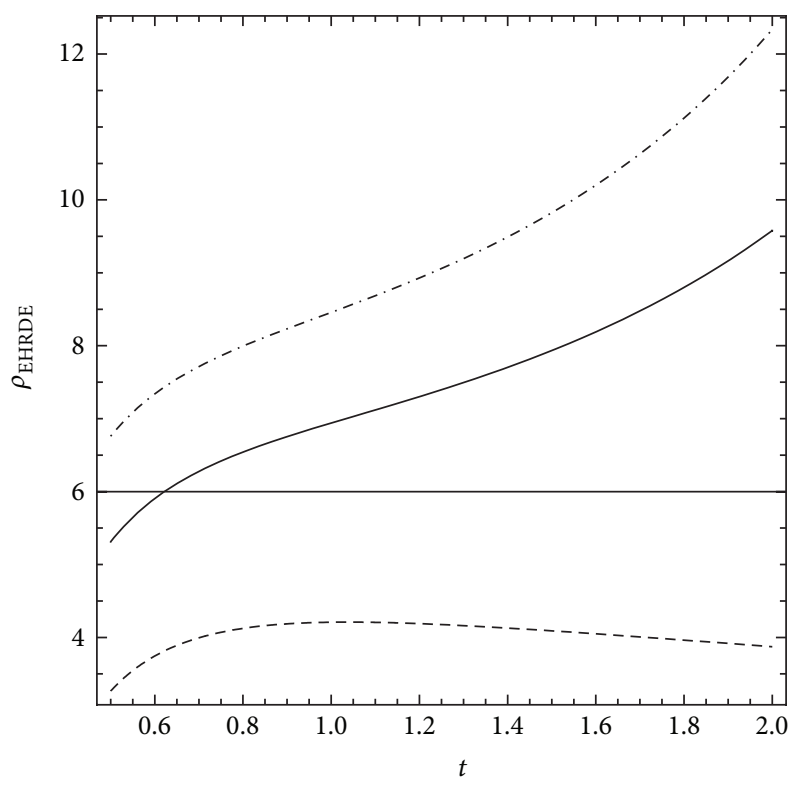

FIGURE 6: Density of EHRDE in fractal universe.

are plotted, respectively, in Figures 9 and 10. In Figure 9, we observe that $w_{\text {MHRDE }}>-1$ for all combinations of $\xi$ and $\eta$. This indicates a quintessence-like behavior. In Figure 10, we observe that $w_{\mathrm{EHRDE}}$ transits from $>-1$ to $<-1$ for $(\xi=1, \eta=2)$ and $(\xi=1.05, \eta=2.01)$ at $t \approx 1.3$. This indicates a transition from quintessence to phantom. Thus, the EoS parameter has a quintom-like behavior in these two cases. For $(\xi=1.01, \eta=2)$, we have $w_{\mathrm{EHRDE}}>-1$.

The deceleration parameter $q=(1 / 2)(1+3 p / \rho)$ for the two models has been plotted in Figures 11 and 12, and in both cases it stays at a negative level, which indicates accelerated expansion of the universe.

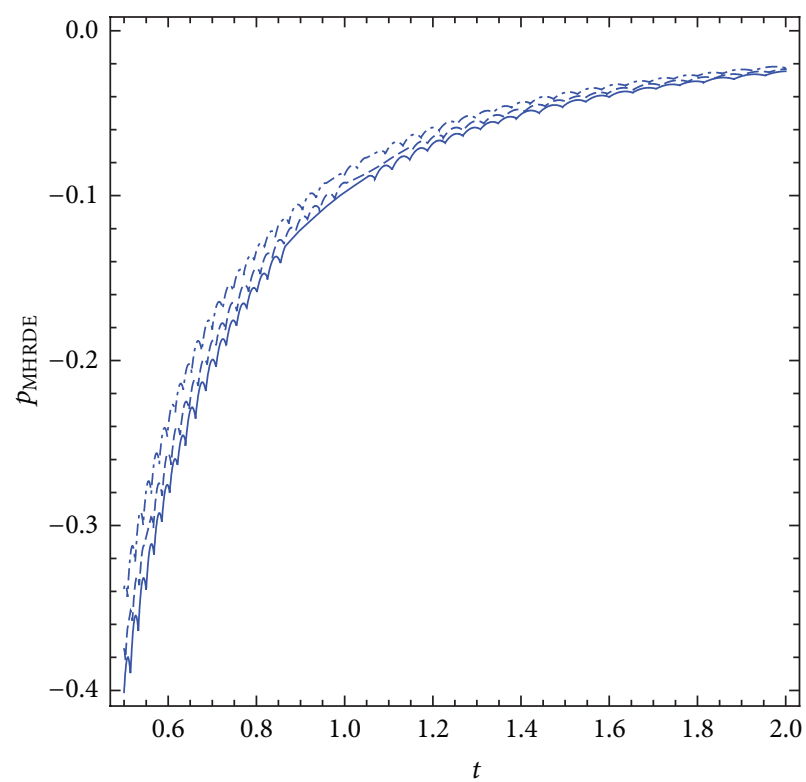

FiguRE 7: Pressure of MHRDE in fractal universe.

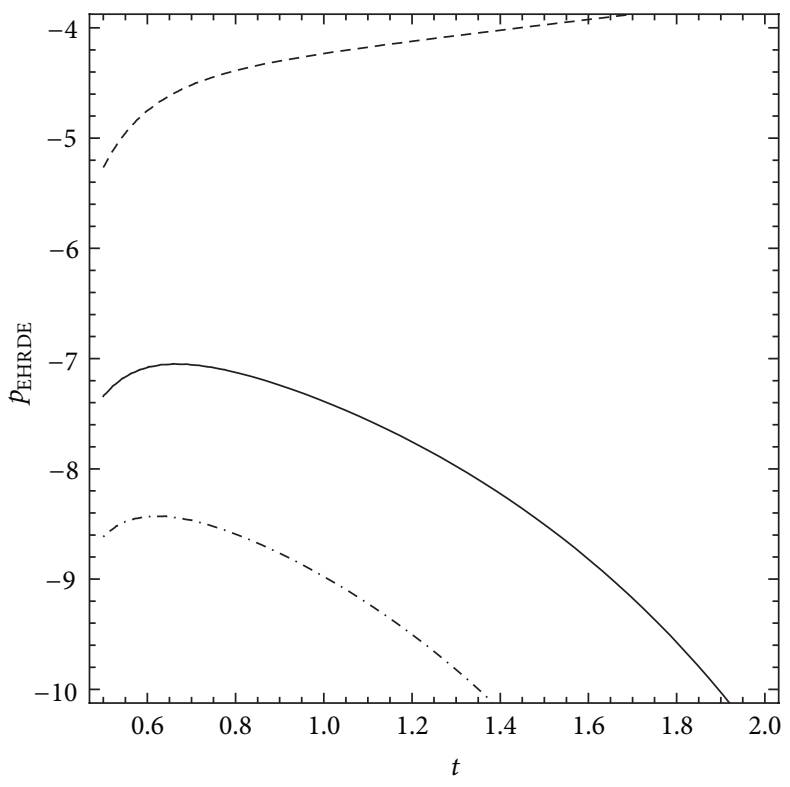

FIGURE 8: Pressure of EHRDE in fractal universe.

\section{Conclusion}

In the present work, we have considered, modified, and extended holographic Ricci dark energy in fractal universe. We have assumed a time-like fractal profile $v=t^{-\beta}$, where $\beta=4(1-\alpha)$. We have reconstructed Hubble parameter for both of the dark energy candidates in fractal universe. Under the reconstructed Hubble parameters, we have reconstructed dark energy candidates in fractal universe, and we have observed that the modified holographic dark energy density is decreasing with cosmic time $t$, and extended holographic dark energy density is increasing with time. The equation of state parameter for modified holographic dark energy has 


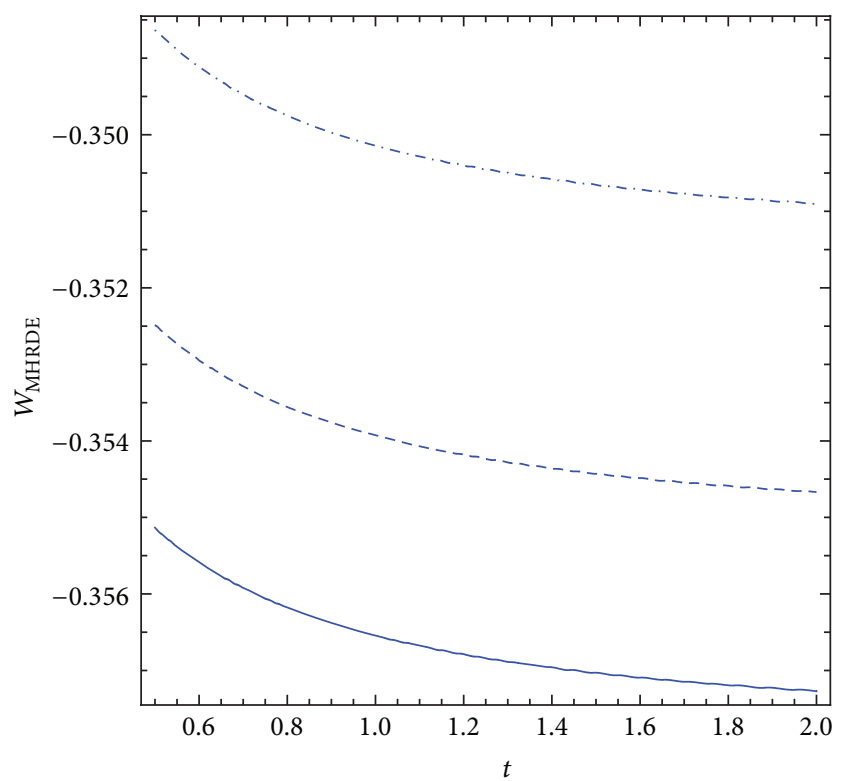

FIgURE 9: EoS parameter for MHRDE in fractal universe.

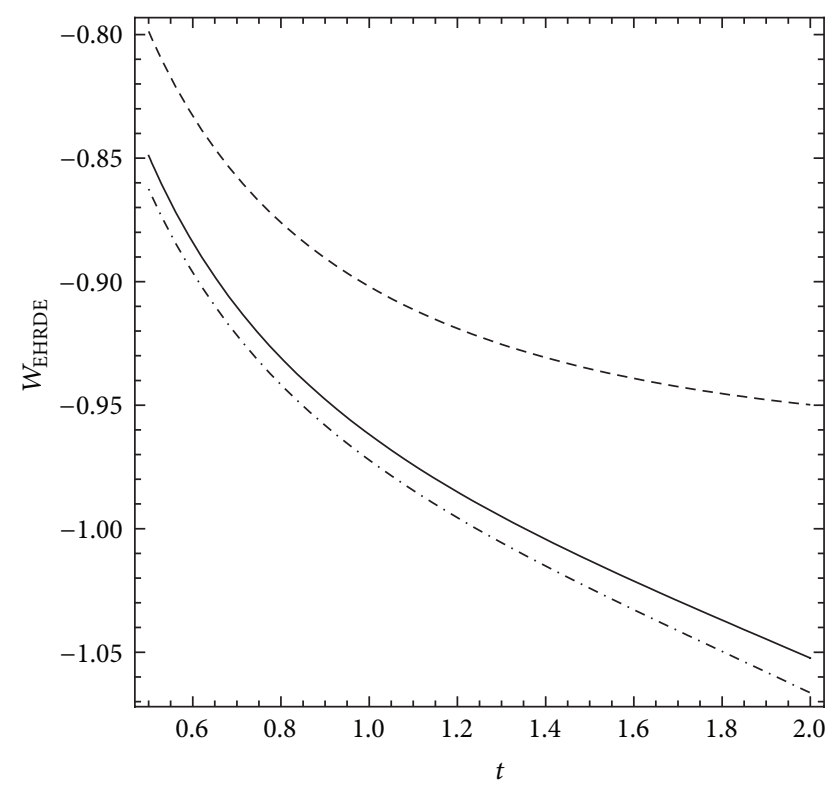

FIGURE 10: EoS parameter for EHRDE in fractal universe.

a quintessence-like behavior and for extended holographic dark energy has a quintom-like behavior. We have seen accelerated expansion of the universe through the deceleration parameter.

\section{Acknowledgment}

The first author Surajit Chattopadhyay acknowledges the research Grant under Fast Track Programme for Young Scientists provided by the Department of Science and Technology (DST), Government of India. The Grant number is SR/FTP/PS-167/2011.

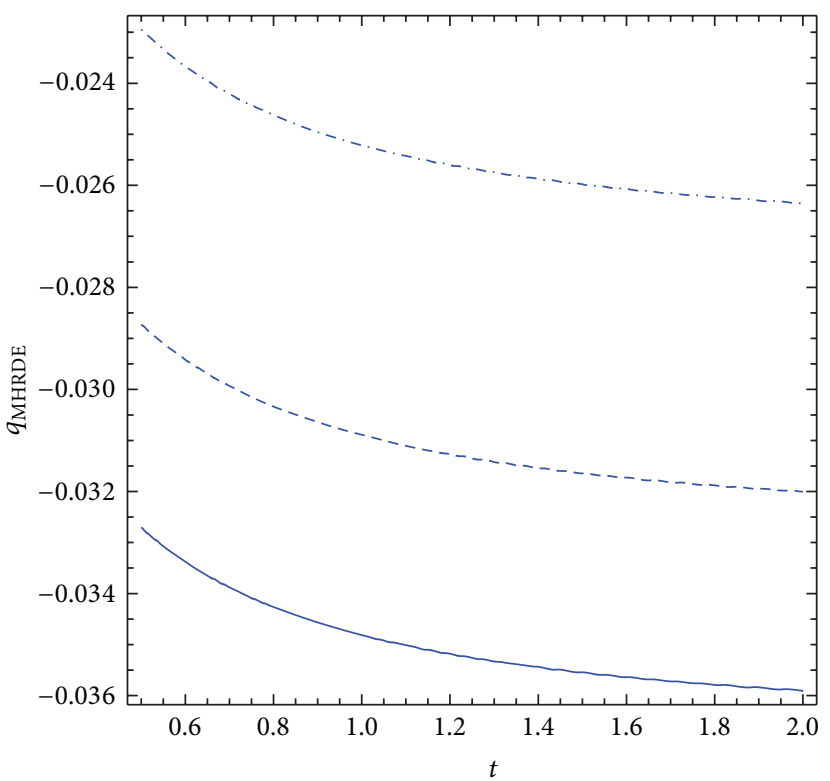

FIgURE 11: Deceleration parameter for MHRDE in fractal universe.

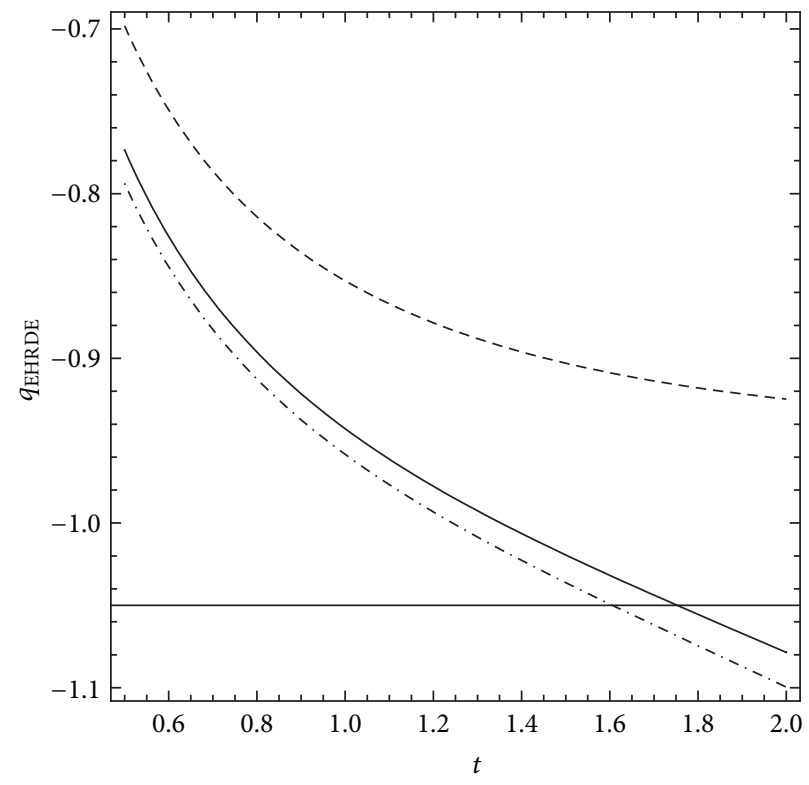

FIGURE 12: Deceleration parameter for EHRDE in fractal universe.

\section{References}

[1] G. Calcagni, "Quantum field theory, gravity and cosmology in a fractal universe," Journal of High Energy Physics, vol. 2010, article $120,2010$.

[2] G. Calcagni, "Fractal universe and quantum gravity," Physical Review Letters, vol. 104, Article ID 251301, 4 pages, 2010.

[3] O. A. Lemets and D. A. Yerokhin, "Interacting dark energy models in fractal cosmology," http://arxiv.org/abs/1202.3457.

[4] A. Sheykhi, Z. Teimoori, and B. Wang, "Thermodynamics of fractal universe," Physics Letters B, vol. 718, pp. 1203-1207, 2013.

[5] K. Karami, M. Jamil, S. Ghaffari, and K. Fahimi, "Holographic, new agegraphic, and ghost dark energy models in fractal 
cosmology," Canadian Journal of Physics, vol. 91, no. 10, pp. 770776, 2013.

[6] M. Li, "A model of holographic dark energy", Physics Letters B, vol. 603, no. 1-2, pp. 1-5, 2004.

[7] R. Bousso, “The holographic principle," Reviews of Modern Physics, vol. 74, no. 3, pp. 825-874, 2002.

[8] L. P. Chimento, M. Forte, and M. G. Richarte, "Modified holographic Ricci dark energy coupled to interacting dark matter and a non-interacting baryonic component," The European Physical Journal C, vol. 73, p. 2285, 2013.

[9] S. D. H. Hsu, "Entropy bounds and dark energy," Physics Letters B, vol. 594, pp. 13-16, 2004.

[10] L. P. Chimento and M. G. Richarte, "Interacting dark matter and modified holographic Ricci dark energy induce a relaxed Chaplygin gas," Physical Review D, vol. 84, no. 12, Article ID 123507, 8 pages, 201.

[11] L. P. Chimento and M. G. Richarte, "Interacting dark matter and modified holographic Ricci dark energy plus a noninteracting cosmic component," Physical Review D, vol. 85, no. 12, Article ID 127301, 4 pages, 2012.

[12] L. N. Granda and A. Oliveros, "New infrared cut-off for the holographic scalar fields models of dark energy," Physics Letters B, vol. 671, no. 2, pp. 199-202, 2009.

[13] M. R. Setare and M. Jamil, "Holographic dark energy with varying gravitational constant in Hořava-Lifshitz cosmology," Journal of Cosmology and Astroparticle Physics, vol. 2010, article 010, 2010.

[14] M. Jamil, M. U. Farooq, and M. A. Rashid, "Generalized holographic dark energy model," European Physical Journal C, vol. 61, no. 3, pp. 471-476, 2009.

[15] A. Sheykhi and M. Jamil, "Power-Law entropy corrected holographic dark energy model," General Relativity and Gravitation, vol. 43, no. 10, pp. 2661-2672, 2011.

[16] M. Jamil and M. U. Farooq, "Interacting holographic dark energy with logarithmic correction," Journal of Cosmology and Astroparticle Physics, vol. 2010, no. 3, article 001, 2010.

[17] M. R. Setare, "Holographic modified gravity," International Journal of Modern Physics D, vol. 17, no. 12, pp. 2219-2228, 2008.

[18] M. R. Setare, "Interacting holographic dark energy in the scalar Gauss-Bonnet gravity," Chinese Physics Letters, vol. 26, no. 2, Article ID 029501, 2009.

[19] C. Gao, X. Chen, and Y. G. Shen, "Holographic dark energy model from Ricci scalar curvature," Physical Review D, vol. 79, Article ID 043511, 7 pages, 2009.

[20] C.-J. Feng, "Statefinder diagnosis for Ricci dark energy," Physics Letters B, vol. 670, pp. 231-234, 2008.

[21] A. Pasqua, A. Khodam-Mohammadi, M. Jamil, and R. Myrzakulov, "Interacting Ricci dark energy with logarithmic correction," Astrophysics and Space Science, vol. 340, no. 1, pp. 199-208, 2012.

[22] A. Pasqua, M. Jamil, R. Myrzakulov, and B. Majeed, "Powerlaw entropy-corrected Ricci dark energy and dynamics of scalar fields," Physica Scripta, vol. 86, no. 4, Article ID 045004, 2012.

[23] A. Pasqua, "Power-law and logarithmic entropy-corrected Ricci viscous dark energy and dynamics of scalar fields," Astrophysics and Space Science, vol. 346, no. 2, pp. 531-543, 2013.

[24] S. Chattopadhyay and A. Pasqua, "Various aspects of interacting modified holographic Ricci dark energy," Indian Journal of Physics, vol. 87, no. 10, pp. 1053-1057, 2013.
[25] A. Pasqua and I. Khomenko, "Interacting Ricci logarithmic entropy-corrected holographic dark energy in Brans-Dicke cosmology," International Journal of Theoretical Physics, vol. 52, no. 11, pp. 3981-3993, 2013.

[26] S. del Campo, J. C. Fabris, R. Herrera, and W. Zimdahl, "Cosmology with Ricci dark energy," Physical Review D, vol. 87, no. 12, Article ID 123002, 14 pages, 2013.

[27] J. G. Silva and A. F. Santos, "Ricci dark energy in Chern-Simons modified gravity," The European Physical Journal C, vol. 73, article 2500, 2013.

[28] S. Chattopadhyay, "Generalized Ricci dark energy in HoravaLifshitz gravity," The European Physical Journal Plus, vol. 127, p. 16, 2012.

[29] L. Xu, W. Li, and J. Lu, "Cosmic constraint on Ricci dark energy model," Modern Physics Letters A, vol. 24, no. 17, p. 1355, 2009.

[30] K. Y. Kim, H. W. Lee, and Y. S. Myung, "On the Ricci dark energy model," General Relativity and Gravitation, vol. 43, no. 4, pp. 1095-1101, 2011.

[31] X. Zhang, "Holographic Ricci dark energy: current observational constraints, quintom feature, and the reconstruction of scalar-field dark energy," Physical Review D, vol. 79, Article ID 103509, 12 pages, 2009.

[32] L. N. Granda and A. Oliveros, "Infrared cut-off proposal for the holographic density," Physics Letters B, vol. 669, no. 5, pp. 275277, 2008.

[33] A. Pasqua and S. Chattopadhyay, "A study on the modified holographic Ricci dark energy in logarithmic $f(T)$ gravity," Canadian Journal of Physics, vol. 91, p. 351, 2013.

[34] Y. U. Fei and Z. Jing-Fei, "Statefinder diagnosis for the extended holographic Ricci dark energy model without and with interaction," Communications in Theoretical Physics, vol. 59, p. 243, 2013. 

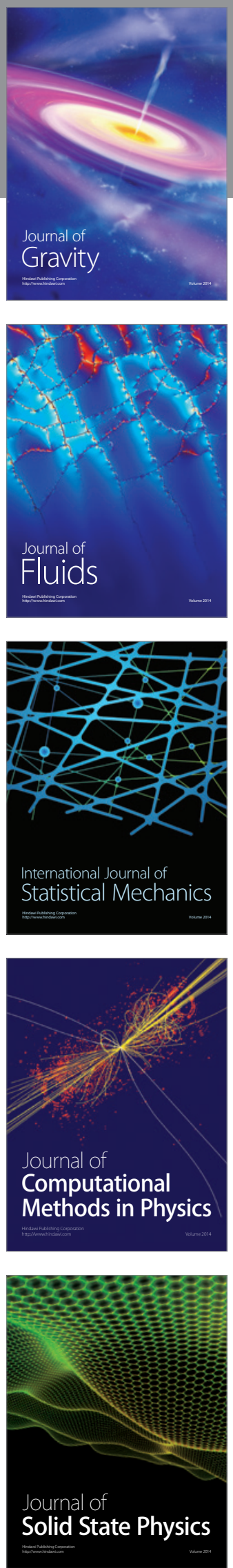

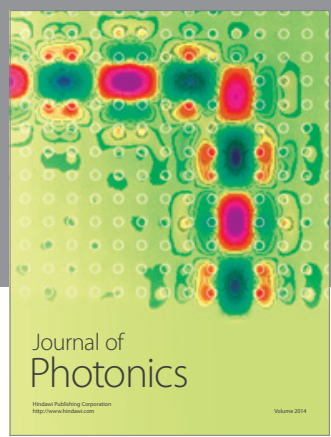

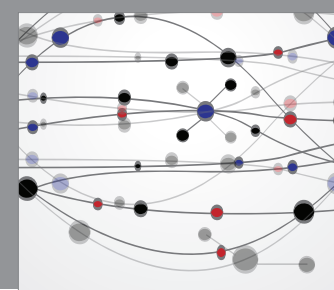

The Scientific World Journal

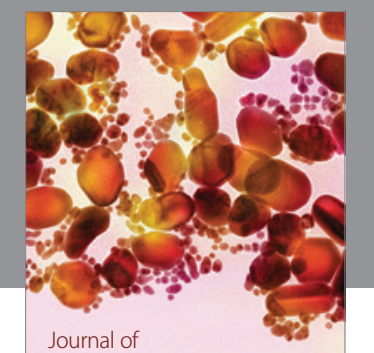

Soft Matter
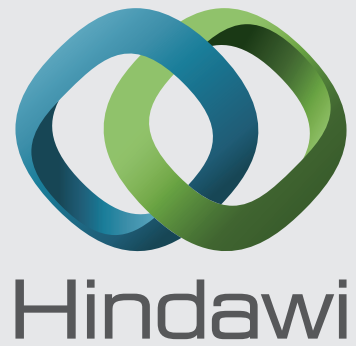

Submit your manuscripts at

http://www.hindawi.com
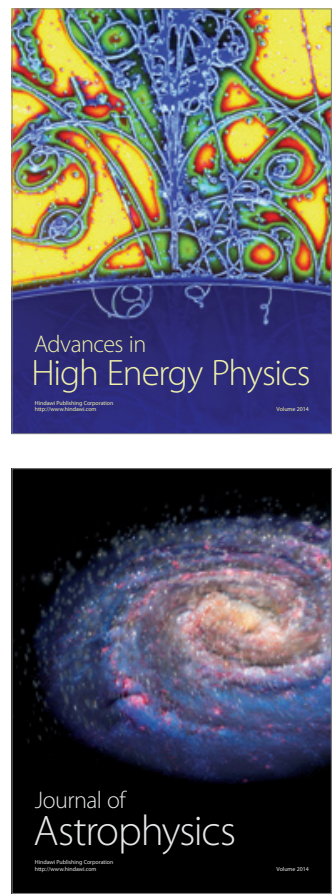
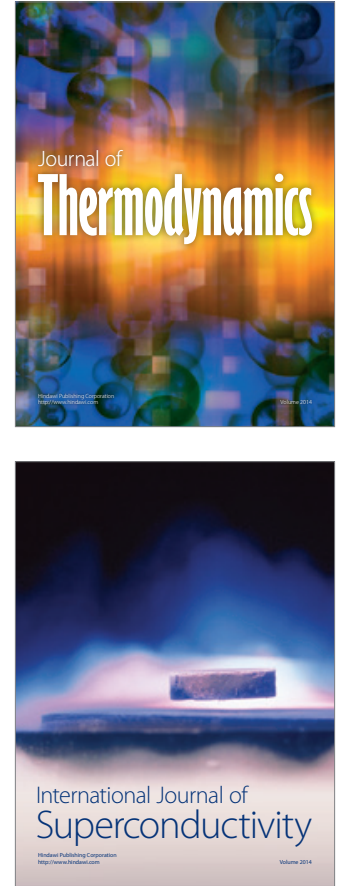
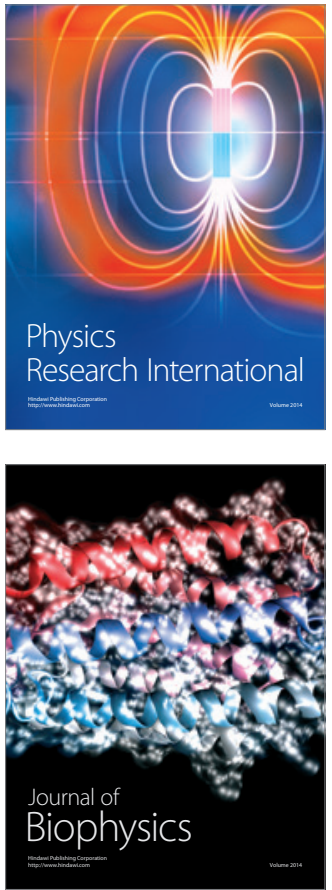
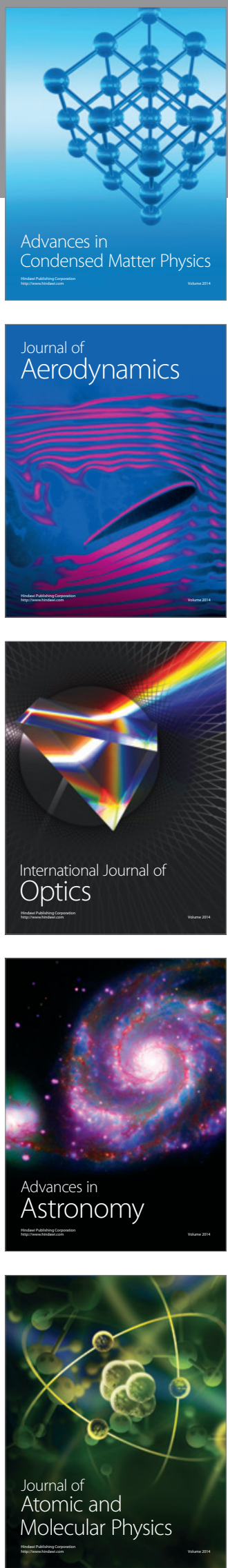